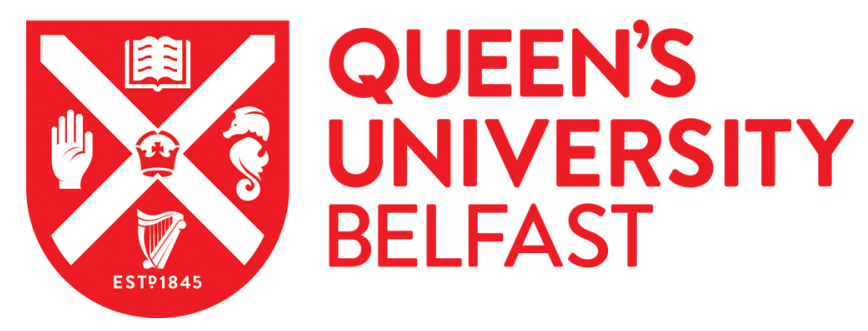

\title{
Spliceosome mutations are common in persons with myeloproliferative neoplasm-associated myelofibrosis with RBC- transfusion-dependence and correlate with response to pomalidomide
} for the RESUME trialists (2020). Spliceosome mutations are common in persons with myeloproliferative neoplasm-associated myelofibrosis with RBC-transfusion-dependence and correlate with response to pomalidomide. Leukemia. https://doi.org/10.1038/s41375-020-0979-6, https://doi.org/10.1038/s41375-020-09796

Published in:

Leukemia

Document Version:

Publisher's PDF, also known as Version of record

Queen's University Belfast - Research Portal:

Link to publication record in Queen's University Belfast Research Portal

Publisher rights

() 2020 Springer Nature Limited.

This work is made available online in accordance with the publisher's policies. Please refer to any applicable terms of use of the publisher.

\section{General rights}

Copyright for the publications made accessible via the Queen's University Belfast Research Portal is retained by the author(s) and / or other copyright owners and it is a condition of accessing these publications that users recognise and abide by the legal requirements associated with these rights.

Take down policy

The Research Portal is Queen's institutional repository that provides access to Queen's research output. Every effort has been made to ensure that content in the Research Portal does not infringe any person's rights, or applicable UK laws. If you discover content in the

Research Portal that you believe breaches copyright or violates any law, please contact openaccess@qub.ac.uk. 


\section{Spliceosome mutations are common in persons with myeloproliferative}

neoplasm-associated myelofibrosis with RBC-transfusion-dependence and correlate with response to pomalidomide

Onima Chowdhury ${ }^{1}$, Jennifer O'Sullivan ${ }^{1}$, Nikolas Barkas ${ }^{1}$, Guanlin Wang ${ }^{1}$, Gemma Buck ${ }^{1}$, Angela Hamblin ${ }^{1}$, Ayalew Tefferi ${ }^{2}$, Haifa K. Al-Ali ${ }^{3}$, Giovanni Barosi ${ }^{4}$, Timothy Devos ${ }^{5}$, Heinz Gisslinger $^{6}$, Qian Jiang ${ }^{7}$, Jean-Jacques Kiladjian ${ }^{8}$, Ruben Mesa ${ }^{9}$, Francesco Passamonti ${ }^{10}$, Vincent Ribrag ${ }^{11}$, Gary Schiller ${ }^{12}$, Alessandro M. Vannucchi ${ }^{13}$, Daobin Zhou ${ }^{14}$, Mary Frances McMullin ${ }^{15}$, Jim Zhong ${ }^{16}$, Robert Peter Gale ${ }^{16}$, Adam J. Mead ${ }^{1}$ for the RESUME trialists

${ }^{1}$ NIHR Biomedical Research Centre and MRC Molecular Haematology Unit, Weatherall Institute of Molecular Medicine, University of Oxford, Oxford, UK

${ }^{2}$ Mayo Clinic, Rochester, MN, USA

${ }^{3}$ Universitätsklinikum Halle, Halle, Germany

${ }^{4}$ Fondazione IRCCS Policlinico S. Matteo, Pavia, Italy

${ }^{5}$ Universitaire Ziekenhuizen, Leuven, Belgium

${ }^{6}$ Medizinische Universität Wien, Vienna, Austria

${ }^{7}$ Peking University, People's Hospital, Beijing, China

${ }^{8}$ Hôpital Saint-Louis, Paris, France

${ }^{9}$ Mays Cancer Center at UT Health San Antonio MD Anderson, USA

${ }^{10}$ University of Insubria, Varese, Italy

${ }^{11}$ Gustave Roussy, Université Paris-Saclay, Saint-Aubin, France Gustave Roussy, Université Paris-Saclay, Villejuif, France,

${ }^{12}$ David Geffen School of Medicine at UCLA, Division of Hematology and Oncology, Los Angeles, CA, USA

${ }^{13}$ Center for Research and Innovation of Myeloproliferative Neoplasms, AOU Careggi, Department of Experimental and Clinical Medicine, University of Florence, Florence, Italy 


\footnotetext{
${ }^{14}$ Peking Union Medical College Hospital, Beijing, China

${ }^{15}$ Queens University Belfast, UK

${ }^{16}$ Haematology Research Centre, Imperial College London, London, UK
}

Correspondence:

Adam J. Mead

NIHR Biomedical Research Centre and MRC Molecular Haematology Unit

Weatherall Institute of Molecular Medicine

University of Oxford, Oxford, UK

Telephone: +44 1865222489

Email: Adam.mead@imm.ox.ac.uk

Myelo-proliferative neoplasm (MPN)-associated myelofibrosis (PMF) is a clonal disorder characterised by decreased blood cell concentrations, bone marrow fibrosis and a risk of transformation to acute myeloid leukaemia (AML). Persons with this cancer who become RBC-transfusion-dependent have a poor prognosis with limited therapy options to reverse their anemia (1). Most persons with MPN-associated myelofibrosis have driver mutations in JAK2, CALR or MPL resulting in aberrant JAK/STAT signaling (2). Non-driver mutations are common in spliceosome components, epigenetic regulators and notably in $A S X L 1, E Z H 2, I D H 1 / 2, S R S F 2$ and U2AF1Q157, which are associated with adverse outcomes and are termed highmolecular risk mutations $(3,4)$.

RESUME was a randomized, double-blind, parallel group, controlled trial, assessing rates of RBC-transfusion-independence in subjects receiving pomalidomide or 
placebo (5). Although response rates were similar, the study identified some differences between responders in the pomalidomide and placebo cohorts, including some subjects with a sustained response to pomalidomide, not observed in the placebo cohort. We reasoned the mutational landscape of subjects in the RESUME study might correlate with these pomalidomide responses.

Baseline DNA samples with corresponding clinical data were available for 205 of the 252 randomized subjects. Median age was 70 years (range, 44-90 years). Additional subject co-variates are reported and displayed in Table S1. We performed targeted DNA sequencing on samples from the 205 subjects using a Fluidigm Access Array 28 gene panel followed by next generation sequencing. Specific methods and analysis pipelines are described in the Supplement.

485 mutations were detected (Table S2). 198 of the 205 subjects (97\%; 95\% Confidence Interval $[\mathrm{Cl}], 95,99 \%$ ) had a mutation in $\geq 1$ targeted gene. 2 mutations were detected in $40 \%(33,46 \%)$ and $\geq 3$ in $39 \%(32,46 \%$; Figure $1 \mathrm{~A}) .7$ subjects had no detectable mutation. JAK2 $2^{V 617 F}, C A L R$ and MPL mutations were identified in $136(66 \%[59,72 \%]), 29(14 \%[8,19 \%])$ and $14(7 \%[4,11 \%])$ of the subjects (Figure 1A). There was no detectable driver mutation in 26 of the subjects with primary myelofibrosis $(13 \%[8,18 \%]$; Table S1). Non-driver mutations were detected in $81 \%$ of subjects $(75,86 \%)$ with a higher frequency in males $(88 \%$ [83, $93 \%$ ] versus $61 \%$ $[48,74 \%] ; P=0.0001) . \quad 45 \%$ of subjects $(38,52 \%)$ had a spliceosome mutation including U2AF1 (22\% [16, 28\%]); SF3B1 (12\% [8, 16\%]) SRSF2 $(7 \%[3,14 \%])$ and ZRSR2 (8\% [4, 12\%]). More spliceosome mutations were detected in men than women $(52 \%$ [44, 60\%] versus $27 \%$ [15, 38\%]; $P=0.001$; Table S1). Spliceosome 
mutations were mutually exclusive in $85 / 92$ subjects. 7 subjects had $>1$ spliceosome mutation. (Figure S1). Spliceosome mutations were also less common in subjects with prior polycythemia vera $(17 \%[2,32 \%])$ compared to subjects with prior essential thrombocythaemia $(39 \%$ [22, 55\%]) and those with primary myelofibrosis (51\% [43, 59\%]; $P=0.074$ and $P=0.002$; Table S1). Mutations in ASXL1 (30\% [24, 36\%]); TET2 (16\% [10, 20\%]), DNMT3A (5\% [2, 8\%]) and EZH2 (5\% $[2,8 \%])$ were detected at rates similar to those reported $(3,6) .46 \%(40,53 \%)$ of subjects had a high molecular risk mutation. Subjects with JAK2 ${ }^{\mathrm{V} 617 \mathrm{~F}}$ were significantly more likely than subjects with a CALR mutation to have a non-driver mutation $(72 \%[64,79 \%]$ versus. $35 \%[(17,52 \%] P=0.0001)$, a spliceosome mutation $(47 \%[36,53 \%]$ versus. $28 \%[6,36 \%] ; P=0.055)$, in particular a U2AF1 mutation $(26 \%$ $[17,32 \%]$ versus none; $P=0.002)$ and a high molecular risk mutation $(49 \%[41,58 \%]$ versus $24 \%[8,40 \%] ; P=0.014)$. Certain mutations were positively correlated including JAK2 $2^{\mathrm{V} 617 \mathrm{~F}}$ and U2AF1 (Odds Ratio [OR], $2.1[0.9,4.4] ; P=0.07$ ), ASXL1 and EZH2 (OR $4.4[1.3,15.7] ; P=0.022)$ and $S F 3 B 1$ and TP53 $(\mathrm{OR}=5[1.1,22.2]$; $P=0.035 ;$ Figure S1).

Median follow-up was 12 months (range, 11-14 months). 52 subjects died (25\%) including 8 transforming to AML. 1-year survival was $76 \%(70,80 \%)$. There were no significant associations between mutation topography and survival including highrisk mutations (Figure 1B). An exception was TP53 mutation which was associated with a 1 -year leukemia-free survival (LFS) of $47 \%(29,63 \%)$ versus. $76 \%(70,81 \%)$ in those without a TP53 mutation $(P=0.0085$; Figure $1 C)$. 
There was no significant correlation between the probability of becoming RBCtransfusion-independent and driver mutation state in either cohort (Table S1). However, subjects with a non-driver mutation were less likely to achieve a 50 percent decrease in RBC-transfusions $(\mathrm{OR}=0.38[0.18,0.8] ; P=0.01)$. When analysed by treatment arm, this reduction was only seen in subjects receiving pomalidomide $(\mathrm{OR}=0.3[0.1,0.7] ; \mathrm{P}=0.009$; Figure $2 \mathrm{~A})$ but not in those receiving placebo $(\mathrm{OR}=0.9[0.2,3.4] ; \mathrm{P}=0.84 ;$ Figure $2 \mathrm{~A})$. In the entire cohort we detected a correlation between having a spliceosome mutation and a lower probability of becoming RBC-transfusion-independent in all subjects (OR=0.5 $[0.2,1.1] ; P=0.096)$. However, this association was significant only in subjects receiving pomalidomide $(\mathrm{OR}=0.33[0.11,0.94] \mathrm{P}=0.038$ versus $\mathrm{OR}=1.1[0.29,4.3] \mathrm{P}=0.87$; Figure $2 \mathrm{~B}) . \quad$ This association was largely attributable to a lower rate of RBC-transfusion-independence in subjects with a U2AF1 mutation receiving pomalidomide $(\mathrm{OR}=0.1[0.021,1.1]$; $P=0.06$ versus placebo $\mathrm{OR}=0.9[0.2,4.8] ; P=0.89$; Figure $2 \mathrm{~B})$. A similar correlation with receiving pomalidomide was detected when the endpoint was a 50 percent decrease in RBC-transfusions in subjects with a spliceosome mutation (OR=0.35 $[0.15,0.84] ; P=0.018$; versus $\mathrm{OR}=1.2[0.4,3.2] ; P=0.79$; Figure $2 \mathrm{~A})$. No other specific mutation or combination significantly correlated with either response category. Re-calculated RBC-transfusion-independence rates in the pomalidomide and placebo cohorts, censoring subjects with splicing factor mutations, showed no treatment effect on RBC-transfusion-independence rates (22\% [12, 31\%] versus $15 \%$ $[3,27 \% ; P=0.40)$ or 50 percent reduction in RBC-transfusions $(35 \%[34,70 \%]$ versus $32 \%$ [8, 64\%], $P=0.75)$. Censoring for non-driver mutations also did not distinguish transfusion independence rates $24 \%$ [8, 40\%] versus $9 \%$ [7, 26\%], 
$P=0.29$ ) or 50 percent reduction in RBC transfusions $52 \%[34,70 \%]$ versus $36 \%$ [ $64 \%], P=0.39)$.

Although considerable data indicate a correlation between high-risk mutations and survival, we found no such correlation (3). There are some likely explanations. $1^{\text {st }}$, all of our subjects were high-risk with 2 independent adverse risk co-variates, anemia and RBC-transfusion-dependence and 2nd, median follow-up was only 1year. Of note, we detected a correlation between TP53 mutation and LFS. TP53 mutation is not typically classified as a high-risk mutation in MPN-associated myelofibrosis but this may need reconsideration (7).

The conclusion of the RESUME study was there was no significant difference in response rates to pomalidomide and placebo in achieving RBC-transfusionindependence or a 50 percent reduction in RBC-transfusion frequency. This conclusion was perplexing as there were several subjects in the pomalidomide cohort responding to pomalidomide, who lost their response when pomalidomide was stopped and regained their response when pomalidomide was re-started. There were no similar subjects in the placebo cohort. Randomization is intended to balance cohorts for known and unknown (latent) co-variates correlated with outcome(s). When numbers of subjects randomized is small, such as the 252 subjects in the RESUME study, the substantial likelihood of an imbalance for $\geq 1$ covariate is presumed to be encompassed in the $P$-value. However, this adjustment is imperfect. We found a correlation between likelihood of response to pomalidomide and non-driver mutations including spliceosome mutations, a correlation not found in the placebo cohort. However, after censoring for non-driver mutations or 
spliceosome mutations, receiving pomalidomide still had no significant impact on rates of RBC-transfusion-independence or a 50 percent reduction in RBCtransfusions. The study was not powered to detect such difference and these analyses were not pre-specified. Consequently, our conclusions are therefore hypothesis-generating and require validation. Our data also suggest a need for mutation topography analyses and stratification pre-randomization in intervention trials in MPN-associated myelofibrosis.

\section{Conflicts of interest}

None.

\section{Author contributions}

OC designed and analyzed experiments and prepared the typescript; JO performed the statistical analysis and contributed to preparing the typescript. NB and GW performed bioinformatic analysis; GB designed the sequencing panels, processed samples and performed experiments; $\mathrm{AH}$ contributed to the design of the panels and advised on data analyses; AT, HKA, GB, TD, HG, QJ, J-JK, RM, FP, VR, GS, AV, DZ, MFM and JZ contributed samples. RPG supervised the project and helped prepare the typescript. AJM conceived and supervised the project, designed experiments and help prepare the typescript. All authors read and approved the typescript.

\section{Acknowledgements}

Supported in part by a National Institute for Health Research (NIHR) academic clinical lectureship (OC), the John Fell Fund (GW), a Medical Research Council Senior Clinical Fellowship (AJM, MR/I006340/1), a Cancer Research UK (CRUK) 
Senior Cancer Research Fellowship (AJM). RPG acknowledges support from the National Institute of Health Research (NIHR) Biomedical Research Centre funding scheme. Supported in part by an unrestricted educational grant from Celgene Corp.

\section{References}

1. Tefferi A. Anemia in myelofibrosis-prevalence, the U2AF1 connection, new treatments. Blood Cancer J. 2017;7(12):648.

2. Rampal R, Al-Shahrour F, Abdel-Wahab O, Patel JP, Brunel JP, Mermel CH, et al. Integrated genomic analysis illustrates the central role of JAK-STAT pathway activation in myeloproliferative neoplasm pathogenesis. Blood. 2014;123(22):e123-33.

3. Vannucchi AM, Lasho TL, Guglielmelli P, Biamonte F, Pardanani A, Pereira A, et al. Mutations and prognosis in primary myelofibrosis. Leukemia. 2013;27:1861.

4. Tefferi A, M. Finke C, Lasho T, A. Hanson C, Ketterling R, Gangat N, et al. U2AF1 mutation types in primary myelofibrosis: phenotypic and prognostic distinctions. Leukemia. 2018;32.

5. Tefferi A, Al-Ali HK, Barosi G, Devos T, Gisslinger H, Jiang Q, et al. A randomized study of pomalidomide vs placebo in persons with myeloproliferative neoplasm-associated myelofibrosis and RBC-transfusion dependence. Leukemia. 2017;31(5):1252.

6. Tefferi A, Lasho TL, Finke CM, Elala Y, Hanson CA, Ketterling RP, et al. Targeted deep sequencing in primary myelofibrosis. Blood Adv. 2016;1(2):105-11.

7. Tefferi A, Guglielmelli P, Nicolosi M, Mannelli F, Mudireddy M, Bartalucci N, et al. GIPSS: genetically inspired prognostic scoring system for primary myelofibrosis. Leukemia. 2018;32(7):1631-42. 


\section{Figure Legends}

\section{Figure 1. The mutational landscape of transfusion dependent myelofibrosis}

(A)Frequency of mutations detected at baseline by targeted gene sequencing $(n=$ 205 patients). Each column represents an individual patient. Only genes with pathogenic mutations detected in at least 5 patients are shown. Each colour corresponds to mutation type. VUS=variant of uncertain significance (see methods). (B) Kaplan-Meier curve of leukemia-free survival (LFS) stratified by HMR at 1-year; HMR did not influence 12-month LFS; HMR-mutated (71\% [95\% Cl 63-78\%]) versus HMR-wild type (WT) patients $(78 \%$ [95\% Cl 71-84\%]), $p=0.13$. (C) Kaplan-Meier curve of LFS stratified by TP53 at 1-year showing a poorer LFS in TP53-mutated patients $(47 \%$ [95\% Cl 29-63\%]) versus TP53-WT (76\% [95\% Cl 70-81\%]) $\mathrm{p}=0.0085$. HMR (high molecular risk - ASXL1, EZH2, SRSF2 U2AF1 Q157)

\section{Figure 2. Impact of mutational status on transfusion responses}

(A) Forest plot depicting influence of mutational status on achievement of $50 \%$ reduction in red blood cell transfusion requirement; patients with SF mutations were less likely to achieve this in the POM arm only $(\mathrm{OR}=0.35[0.15,0.84] ; P=0.018)$. This association with poorer transfusion responses in SF-mutated patients was restricted to POM-treated patients. (B) Forest plot depicting the influence of mutational status on achievement of red blood cell transfusion independence (RBC-TI) by treatment arm; patients with SF mutations were less likely to achieve RBC-TI in the POM arm 
$(\mathrm{OR}=0.33[0.11,0.94] \mathrm{P}=0.038)$ but not in the placebo arm; $\mathrm{OR}=1.1[0.29,4.3]$ $\mathrm{P}=0.87$ predominantly attributable to patients with $U 2 A F 1$ mutations; OR=0.1 $[0.021$, 1.1]; $P=0.06)$. Univariate logistic regression was performed for each group and outcomes with significant $(p<0.05)$ odd ratios $(O R)$ (denoted by *) were adjusted for male gender which was independently significant on univariate analysis. 